\title{
The free-volume structure of a polymer melt, poly(vinyl methylether) from molecular dynamics simulations and cavity analysis
}

\author{
Dušan Račko, ${ }^{1,2, a)}$ Sara Capponi, ${ }^{1,3}$ Fernando Alvarez, ${ }^{3,4}$ Juan Colmenero, ${ }^{1,3,4}$ \\ and Josef Bartoš ${ }^{2}$ \\ ${ }^{1}$ Donostia International Physics Centre, Paseo Manuel de Lardizabal 4, 20018 San Sebastián, Spain \\ ${ }^{2}$ Polymer Institute, Slovak Academy of Sciences, 84236 Bratislava, Slovakia \\ ${ }^{3}$ Departamento de Física de Materiales, Universidad del País Vasco (UPV/EHU), \\ Apartado 1072, 20080 San Sebastián, Spain \\ ${ }^{4}$ Materials Physics Center (MPC), Centro de Física de Materiales (CSIC-UPV), \\ Apartado 1072, 20080 San Sebastián, Spain
}

(Received 6 January 2009; accepted 12 July 2009; published online 10 August 2009)

\begin{abstract}
In this work we analyze and compare the free volume of a polymer system poly(vinyl methylether) (PVME) at $300 \mathrm{~K}$ obtained by the two direct but different approaches: Positron annihilation lifetime spectroscopy (PALS) and computer simulations. The free volume is calculated from the simulated cells of PVME by means of numerical methods based on grid scanning and probing the structure with a probe of a given radius $R_{P}$. The free-volume structure was found to be percolated for small probes at $R_{P}=0.53 \AA$. As the probe radius increases, the cavity structure breaks into isolated cavities, reaching a maximum of the cavity number at $R_{P}=0.78 \AA$. We further develop methods for a geometrical analysis of the free-volume cavities by considering their shape. The geometrical computations show that the cavities have elongated shape with side-to-length ratio corresponding to approximately 1:0.55 and with an average length of $6 \AA$. Based on the overlap between the computed cavities and simplified geometrical representations, the best match of the cavity shape is obtained for the approximation to the ellipsoidal shape (overlap on 84.4\%). A match with other examined shapes follows the sequence: ellipsoid $>$ cylinder $>$ bar $>$ sphere $>$ cube. Finally, the computed geometrical parameters are used as input parameters into the quantum-mechanical models for the orthopositronium (o-Ps) lifetime in various free-volume hole geometries. Comparison with the experimental data gives support for two ideas about the existence of an o-Ps particle in the polymeric matrix: (i) the positronium cannot localize in a portion of very small cavities; (ii) and in the case of the percolated cavities, several o-Ps particles occupy some subcavities in the same cavity. Additionally, radial distribution functions of the free volume indicate the existence of two kinds of free volume, a structured one, corresponding to interstitial spaces along the polymer chain, and the so-called "bulk free volume," distributed randomly in the structure. PALS measurements seem to be mainly related with this bulk free volume. The cavities represented by the idealized geometries are visualized in three-dimensional space providing a unique representation on the free-volume structures. (C) 2009 American Institute of Physics. [DOI: 10.1063/1.3193727]
\end{abstract}

\section{INTRODUCTION}

The "free volume" is rather old and very intuitive, and thus appealing, an idea for describing structural disorder and motions of entities in dense liquids. The free-volume concept has also been used trying to understand and predict transport behaviors of permeant molecules in plastic materials and even in biological membranes. The main and simple ingredient behind the so-called "free-volume" theories ${ }^{1-9}$ is that a particle in a dense system can move if it has the space to do so. However, although it has long been recognized that many thermodynamic, dynamic, and transport properties of dense fluids can be rationalized in terms of the space available for molecular motions, the lack of methods to accurately measure or compute free volume-and even the lack of an unequivocal definition of this concept-have limited the devel-

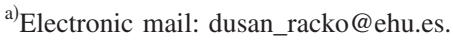

opment of the free-volume theories. Fundamental questions such as: (i) how does the free-volume distribution depend on the molecular structure of the system; (ii) what is the cavity geometry in a particular system; (iii) and what is the role of number of cavities and mean cavity size in transport properties are poorly understood yet.

At present, two methods for a direct determination of the free volume have been developed-the experimental positron annihilation spectroscopy (PALS) and the free-volume calculations from computer simulations. The PALS is based on the annihilation behavior of a physical probe, orthopositronium (o-Ps), in regions of a decreased electron density referred to as "free-volume holes" in condensed matrices. ${ }^{10-16}$ PALS data are used to compute the subnanometer dimensions of the mean free-volume holes in condensed systems based on semiempirical relationships between lifetime of o-Ps and hole size. ${ }^{17-19}$ The first model was derived for a spherical potential as the simplest approximation of the 
free-volume shape. This so-called "standard" model overestimates the actual lifetimes. ${ }^{20}$ Quite recently, several models employing nonspherical hole geometry appeared. Among the first, the annihilation model for the particle in an ellipsoid $^{21-23}$ was derived, followed by models for cuboidal hole shapes. ${ }^{24-27}$ A model which considers a cylindrical approximation for the hole shape has also been derived and used for free-volume studies on syndiotactic polystyrene. ${ }^{28}$ It is noteworthy that the intrinsic drawback of model applications is that additional methods are necessary in order to obtain the parameters characterizing the free-hole shape.

The second approach for a direct characterization of the free volume comes with the atomistic simulations. In this approach the free-volume quantities are obtained by geometrical analyses of the simulated structures obtained by means of the molecular dynamics (MD) or Monte Carlo simulations. Among others, numerical methods based on grid scanning and probing the free-volume structure with a probe of a given radius can be used. These methods produce absolute values of the free volume contained in the structure by means of the volume integration of probe accessible regions determined within probing. ${ }^{29-43}$ A computational method for obtaining the basic geometrical dimensions of a cavity, based on evaluation of the eigenvalues of the gyration tensor radius (at first developed for evaluation of molecular shape), ${ }^{44,45}$ has also been reported and shown the ability to analyze the geometry of the free volume. ${ }^{43}$

Whether or not these two direct free-volume views can be compared or even correlated is still a matter of debate. In fact there are not too many studies carefully comparing results from these two different approaches in the same system and under the same conditions, in particular, in the case of polymer systems. Currently, several attempts to correlate the computed free volume with the free-hole volume calculated from PALS experiments on polymers and glass-forming systems in general can be found in the literature. ${ }^{29,34,38-40,42,46}$ Very recently, some of us have simulated and reproduced the integral quantities of the PALS free volume, such as the mean hole volume and the number density of free-volume holes, over a wide temperature range for two low molecular weight glass-forming systems: glycerol and propyleneglycol. $^{47,48}$ Some other features, e.g., cavity threshold and percolation of the free-volume structure have also been observed, and correlated with PALS results as well as dynamic data. ${ }^{47,48}$

In this work, we compare the free volume obtained from the above mentioned two direct methods in the case of an amorphous polymeric material poly(vinyl methylether) (PVME) at room temperature. First of all, the free volume of a well equilibrated cell of PVME, constructed by means of fully atomistic MD simulations, is considered. The free volume of this sample is analyzed by means of the routines for the numerical free volume already used within the previous free-volume investigations on low molecular weight liquids. ${ }^{47,48}$ Special attention is dedicated to the shape factor of the free-volume cavities. Furthermore, the method originally used for analyzing the characteristic dimensions of cavities $^{43}$ is further developed and included in the computations. By means of this computational method the cavity shape is approximated to certain idealized geometries and the extent of the matching is evaluated. Second, the geometrical information obtained from the computations is used to estimate the o-Ps lifetimes through the existing o-Ps lifetime-to-hole size model relationships and compared to the experimentally measured value. Additionally, the computed free-volume structure has been divided into a distribution of individual subcavities though connected through bottlenecks. We will state that in such a structure an o-Ps particle has more natural conditions for its existence. Based on the comparisons of the computed and measured lifetimes we bring further support for the existence of the so-called cavity threshold. Consistently with the computations on molecular liquids, ${ }^{47,48}$ a portion of small cavities seen by the computer simulation is excluded from the o-Ps particle localization. As we will show at the end of the paper these small cavities might be related to chemical ordering of the polymeric chain. Finally, several unique looks on the computed free-volume structures are provided by visualizations developed for within the aims of work.

\section{METHOD}

\section{A. MD simulations}

The MD simulations were carried out by using the DISCOVER module from Accelrys with the COMPASS forcefield. $^{49-51}$ The system was built by means of the amorphous cell protocol, based on an extension of wellestablished methods for generating bulk disordered systems containing chain molecules in realistic equilibrium conformations. $^{45}$ The initial cubic cell containing seven polymer chains of 100 monomers (7014 atoms) was constructed at $400 \mathrm{~K}$ with periodic boundary conditions. The structure was equilibrated under isobaric-isothermal thermodynamic conditions (NPT ensemble using the isotropic stress tensor constraint) at $400 \mathrm{~K}$ until the conformational distribution, the box side length and the potential energy fluctuated around constant values. In a next step, the obtained structure was optimized by minimizing its potential energy by using standard minimization procedures (Polak-Ribiere conjugated gradients method). Then, the system was dynamically equilibrated by means of a simulation of $1 \mathrm{~ns}$ in the $N V T$ (number of particles, volume, and temperature kept constant) ensemble. This last structure of the dynamic run was used as a starting point for data collection, recording structures every $0.01 \mathrm{ps}$ during MD run of $1 \mathrm{~ns}$. The velocity-Verlet algorithm with a time step of 1 fs was used for the integration of the equations of motion. To control the temperature, instead of a real temperature-bath coupling (Nose-Hoover or Berendsen thermostat) a velocity scaling procedure with a wide temperature window of $10 \mathrm{~K}$ was taken. Under these conditions, greater temperature fluctuations are allowed but the trajectory is disturbed less. It has been shown that by following this simple procedure the results are similar to those obtained with an NVE ensemble, which has the proper Newtonian dynamics. $^{52}$ After the first run, successive runs of $20 \mathrm{~ns}$, collecting data every $0.5 \mathrm{ps}$, were carried out until we gained $40 \mathrm{~ns}$ trajectories without aging phenomena. Starting from this equilibrated cell at $400 \mathrm{~K}$, the temperature was lowered 
down to 375,325 , and finally to $300 \mathrm{~K}$, while the above mentioned procedure was repeated. The system analyzed in this work is that corresponding to $300 \mathrm{~K}$. At this temperature, the PALS data of PVME are already available.

\section{B. The free-volume analysis}

All free-volume data were obtained as average values calculated over 1000 structures sampled from the simulated runs every 1 ps to prevent redundant correlation. Hence $6.5 \times 10^{7} \AA^{3}$ of molecular space have been analyzed. It is worthy of remark, that this procedure means that we are following a "static" approximation in the same way structural magnitudes-as for instance the static structure factor-use to be calculated from simulations. Thereby, temporal evolution of free-volume distributions, i.e., correlations between the free volume at consecutive times, are not here considered.

The free-volume analysis is performed in several consequent steps. First of all, the free-volume regions are determined by the general method of the grid scanning. During this phase, distances of a node of the grid and atoms of the polymer are computed. If the distance for all surrounding atoms is greater than their atomic radius, the coordinates of the node are saved, together with the shortest distance to an atom, according to the algorithm. ${ }^{31}$ The saved information about the shortest distance allows determining the accessible positions for probes with different radii in one single probing run. For a given probe radii the position is accessible only if the saved distance to the nearest atom is larger than the probe radius. Within this investigation the van der Waals radii of atoms were considered. ${ }^{53}$

In the next step the free-volume cavities were built. The cavity construction is based on the investigation of the relative positions of the inserted probes with a given radius. ${ }^{47,48}$ A free-volume cavity is defined as a set of overlapping probes which is detached from other probes, or other cavities, in the simulation box. The construction procedure can be carried out much more efficiently if it is done over subsets of the total amount of the inserted probes. For this reason the cavity construction was performed at first in the probe subsets, similar to the neighbor-lists from the probing procedure. The use of the neighbor-lists, or the subsets with probes respectively, led to considerable savings of the computational time, so the analysis performs around 200 times faster than it would have when analyzing the full structure at once.

Next, the free volume is computed by volume integration of the inserted probes and assigned to the corresponding cavities. The volume integration combines an original fast algorithm, ${ }^{54}$ developed for the calculation of the molecular volume, with the former CAVA algorithm, ${ }^{47}$ which integrates the volume in one loop. The estimated error ${ }^{54}$ of the numerical integration is $0.25 \%$.

Although some computations are made for a set of probes with different radii, the comparison of the mean cavity volume with the PALS experimental value is done only for computations at a typical o-Ps radius, of $R_{P}=0.53 \AA$, ${ }^{11-15}$ which produced the best agreement with experimental data in previous works. ${ }^{47,48}$ In molecular systems with higher spe- cific volume, such as polymers, the free-volume structure (obtained by probing with the small probe) can occur in larger cavities percolated by tiny bottlenecks and pathways. Such "quasicavities" will be distinguished in the simulated structures by means of an additional analysis, based on identifying local maxima of distances between atoms and the probe accessible positions. $^{40}$

The geometrical analysis starts with the calculation of the gyration tensor radius, a diagonalization of which yields eigenvalues corresponding to the semiaxis of the ellipsoid of inertia. $^{45-53}$ For the diagonalization a routine Dsyev.f included in FORTRAN libraries LAPACK 3.0 has been used. ${ }^{55} \mathrm{Cal}-$ culated eigenvectors have been saved with the computed dimensions of the idealized shapes. The coordinate system of the ellipsoid of inertia is already rotated within the routine so that the principal moments of inertia follow the ratio $I_{1}^{2} \leq I_{2}^{2}$ $\leq I_{3}^{2}$. In this way, the third column of the eigenvectors from the routine describes the orientation of the main axis of symmetry of the ellipsoid of inertia. ${ }^{56}$ The angles of rotation necessary, in order to align the cavity with the $z$-axes, can be calculated according the common $z-x-z$ convention of Euler's rotational matrix. ${ }^{66}$ Once the cavities were aligned along the longest axis, overlaps between the computed cavities and volume equivalent shapes (centered on the cavity center of mass) were evaluated. The geometry of idealized shapes, considered here according to the existing PALS models, were cube, sphere, bar, cylinder, and ellipsoid. Additionally, the real length of the wormlike pathways through the cavity could be calculated as an integration of distances between the momentums of the cavity slices, made perpendicularly to the main axis of symmetry. ${ }^{7}$

The MD simulations as well as the free-volume analyses have been performed on a SGI Altix 450 cluster with 16 $\times$ Itanium2 CPU's and 64 Gbyte of memory. The equilibration phase with a production run for one temperature takes around 1 month. The free-volume analyses took approximately 2 weeks of computations when performed in parallel.

\section{RESULTS AND DISCUSSION}

\section{A. The structures and the density}

The first volumetric quantity to be compared with the experimental data is usually the density or the volume of the simulated samples. In MD simulations the density is obtained at the end of the NPT simulation run. A good agreement between the simulated density and its experimental counterpart is the first criterion for the subsequent freevolume studies, since all additional volume of the sample transfers to the free volume, while the volume of the molecular bodies remains almost unchanged with the temperature. This is important especially in simulations of the supercooled region where the PALS free volume is very sensitive to changes of the free volume due to changes of the cavity structure, and because the amount of the free volume itself usually reaches values of a few percent of the total volume. $^{47,48}$ In our case, after the NPT simulation runs, a rather good agreement with the experimental density was obtained. The density of the final structure from the NPT run at $300 \mathrm{~K}$ was $\rho=1.046 \mathrm{~g} \mathrm{~cm}^{-3}$, which agrees well with the 
experimental value ${ }^{58}$ of $\rho=1.053 \mathrm{~g} \mathrm{~cm}^{-3}$ with a deviation of $\delta=0.7 \%$. The resulting cell size was $L=40.1175 \AA$. The satisfactory agreement obtained in the density allowed a direct comparison with the PALS measurements.

\section{B. The cavity numbers and the free volume}

The next volumetric quantity which will be compared with the experiment is the mean cavity volume from MD and CAVA with the mean free-hole volumes from PALS. In this paper we prefer to compare the mean o-Ps lifetimes, which can be directly calculated from the o-Ps lifetime-to-hole size equations, instead of comparing the cavity volumes as we did in previous works. ${ }^{47,48}$ Also in this case the cavity sizes have to be computed at the first step. Based on the cavity volumes and their geometrical characteristics, the corresponding cavity lifetimes are calculated. The mean cavity lifetimes are obtained similarly to the mean cavity volume, as a ratio of the computed lifetime and the number of cavities. ${ }^{59}$ The computed free volume is obtained based on a geometrical analysis of the atomic structures from the MD simulations. Available methods are based on a few distinct principles, allowing calculation of different free-volume features. Note that analytical approaches such as Voronoi tessellation $^{29,60-63}$ and Delaunay triangulation ${ }^{30,64}$ are the most widespread methods for the free-volume analysis and usually come with the standard simulation software. However, these methods evaluate the free-volume features by means of the polyhedrons, rather than by evaluating the absolute amounts of the free volume. Therefore we used a numerical method for the free-volume computation based on a grid-scanning or probing in order to obtain absolute sizes of the free-volume cavities accessible to a probe with a specific geometry. The free-volume analysis usually means a very demanding computational task, due to the geometric complexity of the intermolecular free space. The optimal reduction of the computational expenses have been obtained by employing $7^{3}$ neighbor-lists within the probing and $12^{3}$ neighbor-lists during the cavity construction, while the total computational time necessary has been reduced by 200 times.

The free volume from the probing procedure and the consequent numerical integration typically produces bi- or trimodal distributions of the cavity volume, usually with a large number of very small cavities. ${ }^{29,38,39}$ As suggested previously, these small cavities have to be taken out of consideration in order to reach the experimental values of the mean hole volumes. ${ }^{34,47,48}$ The elimination of the small cavities has a negligible effect on the total amount of the probe accessible volume, but significantly affects the total amount of the cavities. The physical reason for such elimination of the small cavities was given by assuming that such cavities are energetically not suitable for the o-Ps particle localization. ${ }^{65}$ In Fig. 1(a), a distribution of the cavity number as a function of the probe size is shown. All distributions show a similar behavior: Starting from the small probes, the number of cavities is lower, whereas the major part of the free volume is situated in one cavity percolated through the whole freevolume structure. Additionally, a relatively large portion of
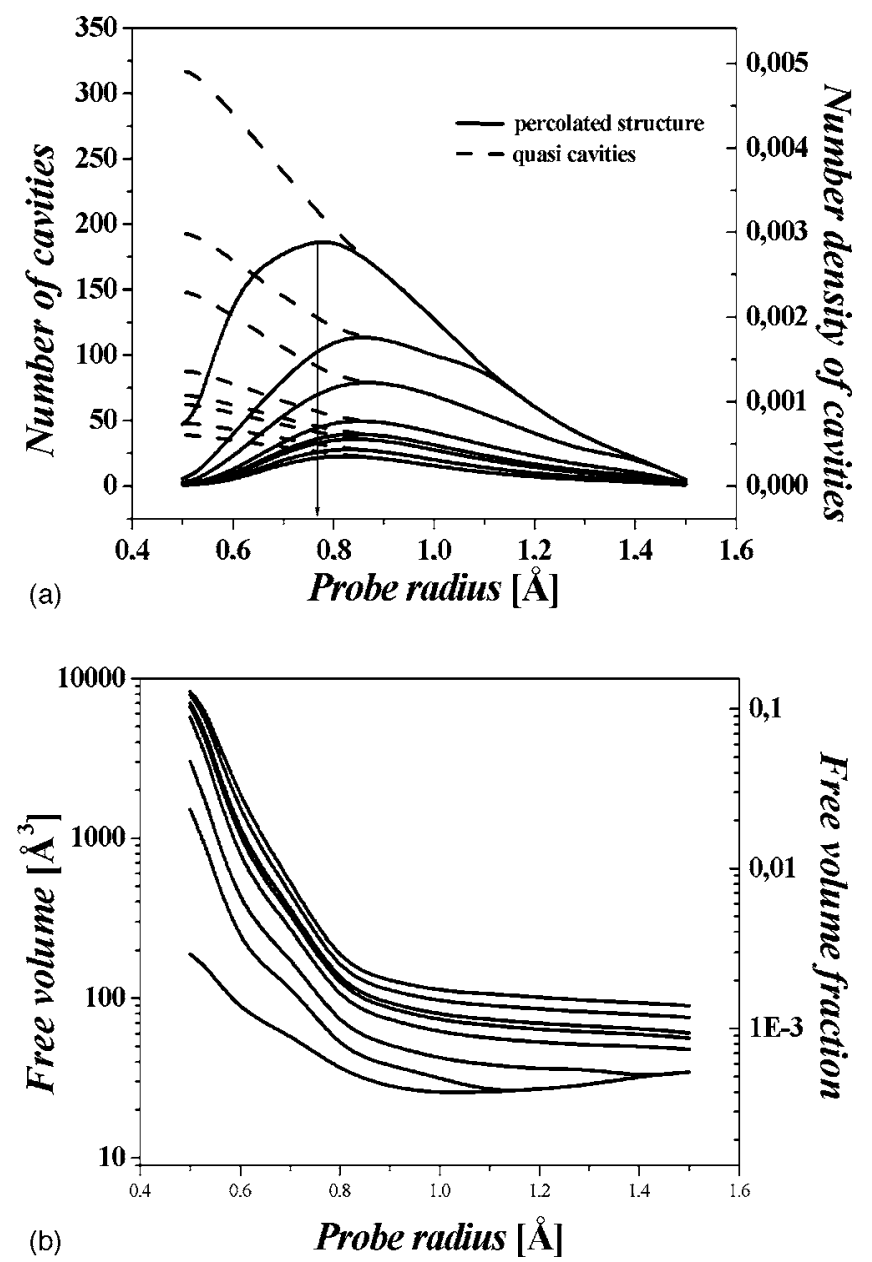

FIG. 1. (a) The cavity number distribution and (b) the total free volumes with different probe radii. The lines correspond to cavities with different volumes, from the top $V_{C}>0,5,10,20,26.5,30,40$, and $50 \AA^{3}$. The dashed lines correspond to a system with a percolated structure divided into subcavities.

small cavities consisting of one single probe can be detected in the free-volume structure. As the probe radius increases, the cavity structure breaks into larger isolated cavities, reaching a maximum at $0.78 \AA$. Beyond this maximum, the total number of cavities decreases again because of the decreasing number of the free-volume regions, large enough to contain a probe of such radii. The position of the peak is close to the radius of the o-Ps probe and may be an illustration of how can the PALS probe be sensitive in detection of a certain accessible part of the free-volume structure. The position of the peak may slightly shift to lower radii when the system becomes denser. In the case of the previously studied glycerol system the position of the peak was found around $0.6 \AA .47$

A computation of the mean cavity volumes on the percolated free-volume structure would bring a rather nonrealistic presumption to the model, i.e., the positronium particle can detect the whole cavity structure. Such calculation would also be strongly biased by the box size effects. Two distinct approaches can be found in the literature for the treatment of the percolated cavities in polymeric systems with higher specific volume. First of all, a set of different probe radii has been examined upon the free-volume studies. ${ }^{34,40,41}$ Here we 
TABLE I. The table shows averaged values of: $A$-asphericity (Ref. 45), $C$-acylindricity (Ref. 45), $a^{\prime} / c$-ratio of the geometric average of minor axes to the major axis, $\varepsilon$-eccentricity (Ref. 21 ), $L$-cavity length, and $L_{\Gamma}$-inertial length (Ref. 43).

\begin{tabular}{lrllllll}
\hline \hline & $A$ & $C$ & $a^{\prime} / c$ & $\varepsilon$ & $\begin{array}{c}L \\
(\AA)\end{array}$ & $\begin{array}{r}L_{I} \\
(\AA)\end{array}$ \\
\hline Number average & 2.064 & 0.461 & 0.689 & 0.724 & 6.041 & 3.317 \\
Volume weighted average & 16.036 & 3.592 & 0.550 & 0.812 & 22.269 & 8.471 \\
\hline \hline
\end{tabular}

show that when the probe of $0.80 \AA$ is used the percolated structure breaks into separate cavities [Fig. 1(a)]. Although in this case the system holds sufficient amount of the larger cavities, with a typical number density of about $1 \times 10^{-3} \AA^{-3},{ }^{11}$ the free volume probed by employing larger probes drops rapidly. Hence, the amount of the free volume is not sufficient to reach the experimental values of the mean hole volume for the probe of radius $R_{P}=0.80 \AA$ [Fig. 1(b)]. Instead, we additionally suppose that the free-volume structure, sampled with a probe of radius $0.53 \AA$, consists of the regions of the raised free-volume regions, i.e., quasicavities linked together by tiny pathways and bottlenecks. In such cases, the cavities are reconstructed around the local maxima of distances calculated between an inserted probe and atoms. ${ }^{40}$ The cavity free volume corresponds to the accessible volume of a probe with the typical o-Ps radius of $0.53 \AA$, while the cavity number of large cavities is similar to that probed by probes at the maximum of the cavity number distribution.

\section{The cavity shape}

In this section we investigate the shape characteristics of the cavities. Here we evaluate the match of cavity shape to five basic geometries: sphere, cube, bar, cylinder, and ellipsoid. The dimensions of these basic geometrical shapes are obtained by using the method for calculating the eigenvalues of the gyration tensor radius of the free-volume cavities. ${ }^{43}$ On the basis of the eigenvalues, the dimensions of the idealized shapes have been calculated in the following way: First of all, a geometrical average $a^{\prime}=\sqrt{a b}$ of the shorter semiaxes and its ratio with the major semiaxis $c$ has been calculated (Table I). Then, the corresponding dimensions have been calculated on an idealized shape with a volume equivalent to the cavity volume, so that the precalculated ratio $a^{\prime} / c$ was maintained. The obtained dimensions of the basic geometrical shapes of cavities will be used as an input parameter to the semiempirical o-Ps lifetime-to-hole size models, approximating the cavities to basic geometries. The computed lifetimes from these models will be used for further comparison with the experimental PALS value of the o-Ps lifetime.

The first indications about the irregularity in the freevolume structure or deviations from the spherical shape of cavities can be inferred by the cavity number distribution shown in Fig. 1(a). In a cavity structure consisting of a polydisperse distribution of cavity volumes with a spherical shape, the distribution would necessarily show a continuous drop when increasing the probe radius. In a monodisperse structure of spherical cavities, the cavity number distribution would abruptly drop to zero, when a probe radii is larger than actual radii of cavities. Only in a system with irregularities about the length scales of the probe, a maximum of the cavity number distribution can be developed. In the following, the cavities are numerically examined in order to check whether they have a characteristic dimension, i.e., if they are flat, elongated or concentric. At first, the principle moments of inertia of the cavities have been calculated. Next, the ratios of the minor axes $a \leq b$ to the major axis of symmetry $c$ have been calculated and histograms have been made upon an analysis of cavities over 1000 structures. Consistently with a previous work on poly(amid imid) ${ }^{43}$ Fig. 2 shows that the cavities have a characteristically elongated shape, but with a large fraction of spherical cavities $\sim 17.5 \%$ sampled by a single probe. The fraction of spherical cavities rises with increasing the probe radius, because of the increasing fraction of the cavities sampled by only one probe. A higher occurrence of the characteristic ratios below the diagonal of the histogram indicates also slightly flat-shaped cavities. The flat shape of the cavities can have its origin in the flat-shaped geometry of polymer segments [see Fig. 3(a) below]. The calculated mean value of the ratio between the major axis
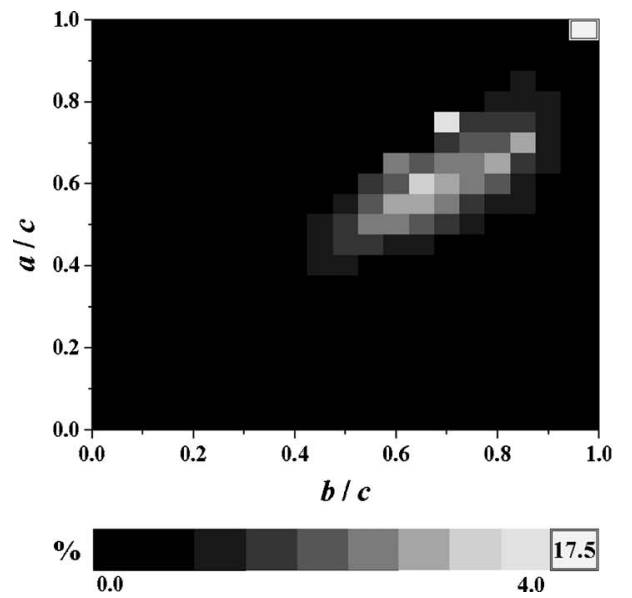
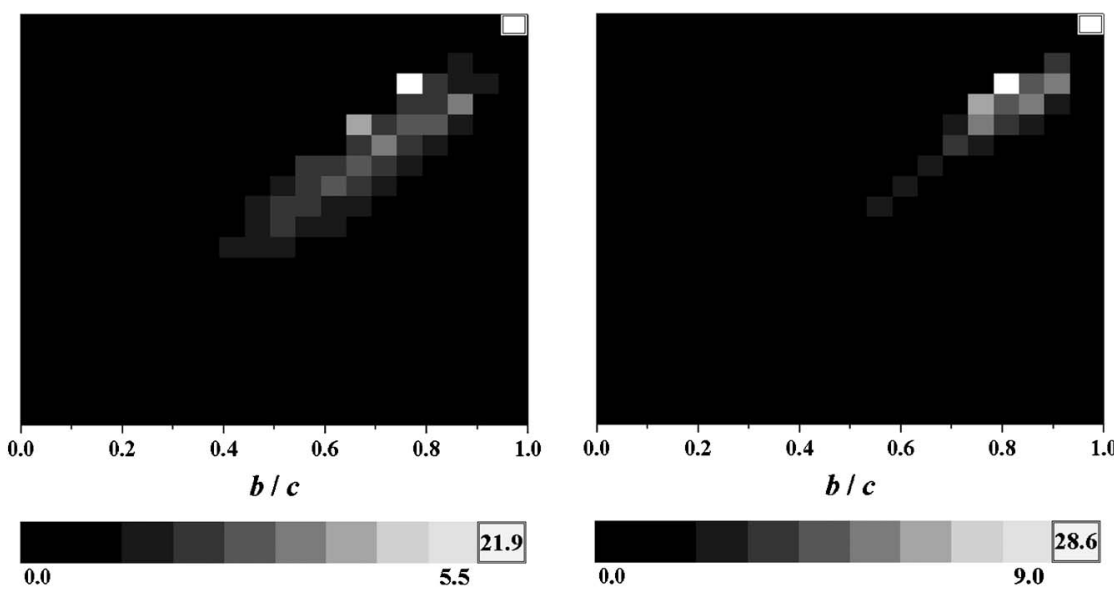

FIG. 2. The characteristic dimension of the cavities. The figure shows a histogram of the cavities, with the calculated minor to major semiaxis ratios, where $a \leq b \leq c$. The histograms were computed for three different probe radii, from the left $R_{P}=0.53 \AA$ (quasicavities), $R_{P}=0.90 \AA$, and $R_{P}=1.50 \AA$. The data are calculated as an average over 1000 structures. 


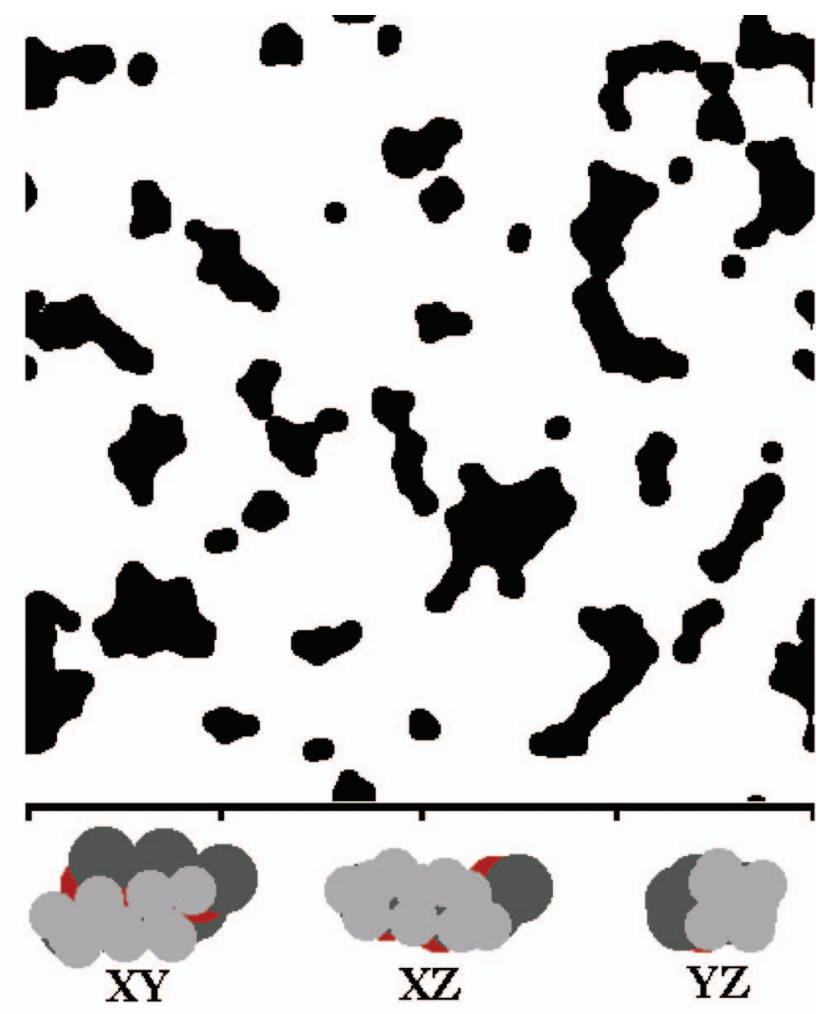

(a)

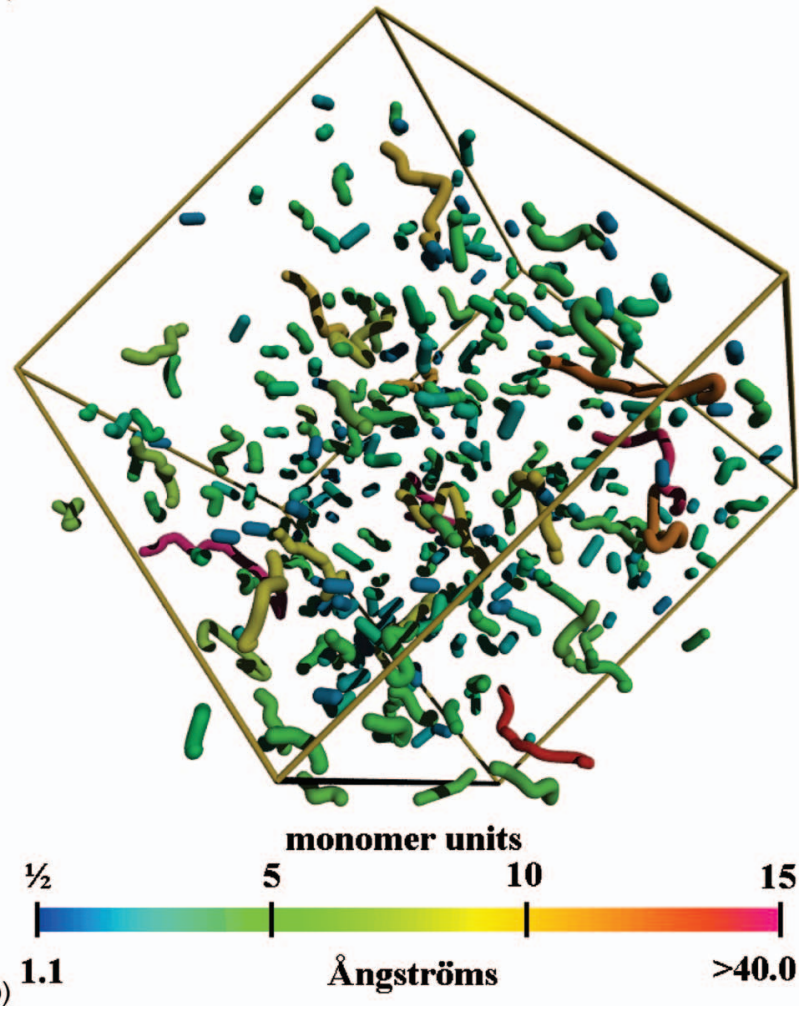

FIG. 3. The free-volume structure computed with a probe with $R_{P}$ $=0.53 \AA$. (a) A slice profile of the free-volume structure obtained by cutting of the simulation box perpendicularly to the $z$-axis (at $10 \AA$ ). Below the picture of a polymer segment consisting of 3 monomers is shown in the same scale for a better comparison. The segment is shown in different views as a projection into $X Y, X Z$, and $Y Z$ planes. (b) The three-dimensional picture shows the wormlike pathways within the cavities. The thickness of the wormlike bodies corresponds to the diameter of o-Ps particle $2 R_{P}$. The different lengths of the cavities are distinguished by the color scale. The length scale is expressed both in angstroms, as well as in monomer-length units. and geometrical average of the minor axes is 1:0.55. The ratio is maintained almost at the same value also for the cavities determined with the larger probes, while it slightly decreases to about 1:0.7 for the largest probes used in our free-volume computations. This is probably due to the fact that a major part of the cavities is formed by a single large probe. The averaged inertial length of the cavities or the length of the major axis of symmetry respectively, is $3.32 \AA$. The averaged integrated length of the wormlike pathways through the cavity is $6.04 \AA$, implying an average tortuosity (defined as the ratio of total path length divided by the endto-end distance) of 1.68 .

Figure 3(a) shows a representative slice profile of the free-volume structure obtained by cutting off the simulation box perpendicularly to the $z$-axis at position $10 \AA$. The cavity lengths typically reach the length of several monomer units, where the monomer length is used as the distance between building units measured on the polymer backbone $\sim 2.5 \AA$. The longest cavity length observed was of the order of 15 monomer units. In the shown cut we can observe that cavities are oriented nonuniformly in various directions. The long cavities, which run along the direction of the slice, substantiate the elongated shape of the cavities. Exceptionally the small cavities, with volume approximating the volume of the o-Ps probe, are mostly spherical. Besides the elongation, the cavities show irregularities or ledges on the length scale comparable with the diameter of the o-Ps particle. Similar cavity branching has been already reported on polymers with ultrahigh free volume. ${ }^{39,40}$ On the other hand, the slice does not show the percolation of the free volume, indicated within the cavity construction. This suggests that the free-volume structure does not percolate through continuous pores but in more complicated fashion by interconnecting individual cavities in three-dimensional space. The connections are most likely tiny cavity pathways and branches. In Fig. 3(b) we can see the overall view on the cavity pathways in the free-volume structure already redistributed into subcavities. These "wormlike" pathways have been obtained by connecting momentums of cavity slices, made perpendicularly on the longest dimension of a cavity. The picture shows that the longer cavities are separated by longer distances. A large number of small cavities occur around the long cavities. Short cavities display almost no tortuosity. In the case of longer cavities the irregularities develop in all three dimensions, resulting into tortuous pathways. Additionally the picture confirms the nonuniform orientational distribution of the cavities.

By evaluating the overlap of the volumes between the computed cavities and the idealized (volume equivalent) shapes, such as sphere, cube, bar, cylinder, and ellipsoid, we obtain a match to a simplified approximation of shape. The computed match of the cavity shape with the idealized geometry, averaged over all cavities, follows the order: ellipsoid $>$ cylinder $>$ bar $>$ sphere $>$ cube (see Table II). As observed earlier, ${ }^{43}$ the deviations from the regular shape increase with the volume of the cavity. As a result, the volume weighted averages are lower with respect to the number averages. Figure 4 shows the free-volume cavities with the simplified geometries and the characteristic dimensions ob- 
TABLE II. The numeric evaluation of the cavity shape approximations. The numbers stand for the match to an idealized geometry in terms of the volume overlap, expressed in percent.

\begin{tabular}{lccccc}
\hline \hline & Cube & Sphere & Bar & Cylinder & Ellipsoid \\
\hline Number average & 67.1 & 72.5 & 78.1 & 81.2 & 84.4 \\
Volume weighted average & 55.7 & 58.8 & 64.6 & 66.3 & 68.5 \\
\hline \hline
\end{tabular}

tained from the numerical analysis. Together with the simplified geometrical representation, two-dimensional (2D) histograms display conditions for the best matching with an idealized shape approximation. Figure 4 shows that the worst approximation of the cavity shape probed by a spherical probe is obtained for the cubic geometry. As expected, also the sphere represents a highly idealized geometry for the real molecular systems giving the spherical geometry the second worst match with the cavity shape. Furthermore, Fig. 4 infers that the flexible geometries, enabled to change ratio between the sides and to elongate, produce a better match with the real cavity shape. We can see that by giving the cubic geometry the freedom to elongate in one direction makes it a better representation than the spherical one. The best match is obtained for the ellipsoidal geometry, which is also mathematically the best spatial position average of the irregular set of probes representing the cavity. The match computed as an average over all cavities is $84.4 \%$ for ellipsoids. Similar match is provided by the cylindrical geometry. As we show in the following text, the semiempirical lifetime-to-volume correlation with the cylindrical geometry of the potentialwell, will be the optimal for the o-Ps lifetime estimate. Additionally, the graphical representations allow considering the cavity size along with cavity shapes and distributions in the volume of the simulation box. We can observe that the structural samples contain large number of small and midsize cavities, with volumes between the probe volume $V_{P}$ (o-Ps) $=0.63 \AA^{3}$ and the volume of a monomer unit $\left(67 \AA^{3}\right)$. Besides hundreds of these small size cavities several large cavities with volume of a few monomer units occur in the structure. The large cavities seem to be separated randomly with correspondingly large distances within the structure. Despite the small cavities with prevailing spherical geometry the larger cavities are more elongated.

\section{The computed free volume and the o-Ps lifetime}

In this section, the computed and experimental free volumes are compared and discussed in terms of the o-Ps lifetimes. The o-Ps lifetimes corresponding to the simulated cavities are calculated by means of the o-Ps lifetime-to-hole size relationships. Because an irregular shape of the free holes can be expected, due to the complex geometry of the molecular bodies and the typical sizes detected by the PALS, which are on the scales of such irregularities, the shape of the holes should be taken into account in the actual lifetime-tohole size relations. Within the comparisons all the five existing models for infinite potential with a basic geometry have been examined. Note that in the case of cuboidal hole shapes with nonequivalent sides the model for the small hole sizes, typical for disordered systems, has been employed. ${ }^{24}$ The
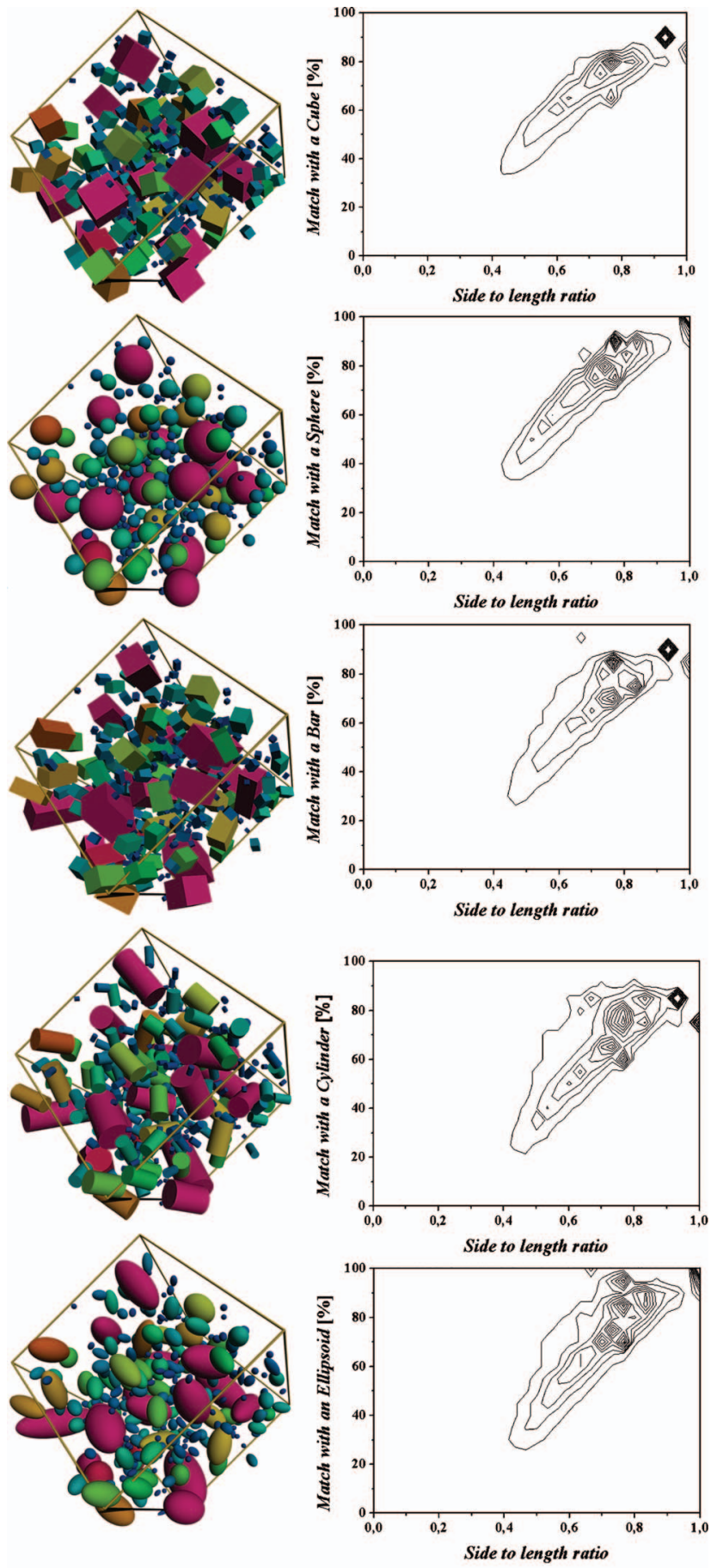

monomer units

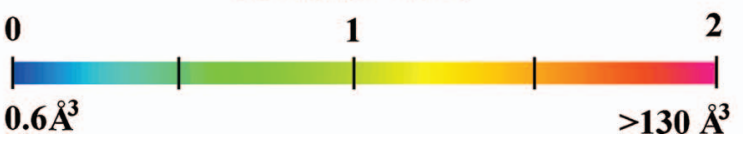

FIG. 4. The evaluation of the match of the shape approximation for several idealized geometries, from the top for cube, sphere, bar, cylinder, and ellipsoid. The cavities in the three-dimensional cavity structure are represented by the simplified geometries (left). The volumes of the cavities are distinguished according to the color scale. The 2D histograms show the map of the highest match occurrence for corresponding geometry (right).

model for cuboidal holes in the size range of mesopores ${ }^{25-27}$ provides negative values of lifetimes corresponding to cube sizes below a few tens $\AA^{3}$. The characteristic dimensions of the idealized geometrical representations of the cavities, 


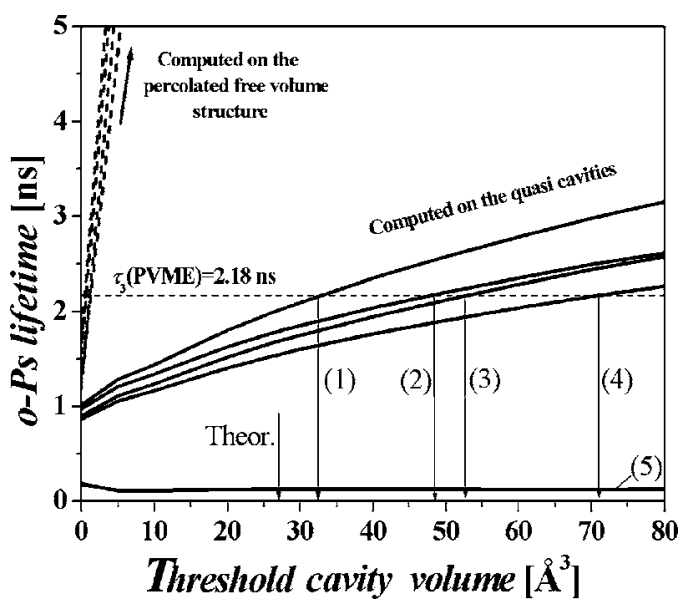

FIG. 5. The mean o-Ps lifetime as a function of the threshold cavity volume, computed by means of the o-Ps lifetime-to-specific shaped hole size relationships with different geometries for (1) sphere (Ref. 19), (2) cylinder (Ref. 28), (3) cube (Ref. 24), (4) bar (Ref. 24), and (5) ellipsoid (Ref. 21), based on the input parameters obtained from the MD simulations and the subsequent cavity analysis. The arrows show an estimated threshold cavity volumes at the experimental value of o-Ps lifetime at $300 \mathrm{~K}$, together with the theoretical value predicted for spherical model (Refs. 34 and 65). The mean o-Ps lifetimes are calculated by using the Eq. (1) for the free-volume structure with subcavities (solid lines), as well as for a percolated structure (dashed lines).

which are necessary as the input parameters to the o-Ps lifetime-to-hole size relationships, have been obtained during the cavity shape analysis (see previous Sec. III C). The mean o-Ps lifetimes of the positronium are calculated according to a modified formula, which we previously used for a calculation of the mean cavity volumes ${ }^{47,48}$

$$
\tau_{3}=\frac{\sum_{V_{c} \geq V_{\lim }} \tau_{c, i}}{\sum_{V_{c} \geq V_{\lim }} N_{c, i}}
$$

where $\tau_{C, i}$ is a calculated lifetime for a cavity $i$ with a given geometry and with a volume $V_{C}$ larger than the threshold cavity volume $V_{\text {lim. }}$. This represents the volume of the smallest cavity of a given shape where an o-Ps particle can still be localized. Below this volume, the cavities would be restricted from o-Ps localization. ${ }^{34,47,48,65}$ The computed mean o-Ps lifetimes are shown in Fig. 5 as a function of the threshold cavity volume and compared to the experimental value of o-Ps lifetime in PVME at $T=300 \mathrm{~K}$, i.e., $\tau_{3}(T)$ $=2.18 \pm 0.04$ ns. ${ }^{66}$ Furthermore, the o-Ps lifetimes were calculated for both, the nonrealistic case of the positronium in a percolated cavity, and the case of the o-Ps particles occupying several regions of an increased free-volume occurrence, i.e., the subcavities of the percolated cavity.

In the first case, we can see that although the experimental mean cavity lifetimes could be attained, the o-Ps would have to exist under extreme conditions. The o-Ps particle has to occupy also the energetically restricted small cavities and at the same time a single positronium particle occurs in the huge free-volume cavity percolating through the whole structure. In the other case, the simulation of the positronium in subcavities represents a more natural picture about the o-Ps particle localization. Moreover, a common value of threshold cavity volume (restricted from the o-Ps localization) is computed. The threshold value for the spherical model ${ }^{34,65}$ is around 26.5 or $30 \AA^{3}$ in simulations. ${ }^{47,48}$ According to the models, ${ }^{21-28}$ the strongest o-Ps lifetime-to-volume correlation occurs when the cavity volume expands in all three dimensions equally. In this way, the o-Ps lifetime calculated for a particular elongated cavity by the models, considering the nonspherical holes, are lower with respect to the spherical model. On the other side, larger cavity volumes can be necessary for a localization of o-Ps particle. The threshold values $V_{\lim }$ estimated for the elongated geometries upon the Fig. 5 are larger being $48 \AA^{3}$ for cylinder, $53 \AA^{3}$ for cube, and $70 \AA^{3}$ for bars. From Fig. 5 we can see that the computed lifetimes for ellipsoid move around values close to zero. The model is limited to larger and axially symmetric ellipsoids with the small eccentricity, lower than $1-(1.656 /(1.656+a))$, where $a$ is the major semiaxis of the ellipsoid. ${ }^{21-24}$ Although the geometry of the ellipsoid approximates the shape of the cavity with the best agreement (Table II), due to the overall ratio of $2: 1$ and the corresponding eccentricity $\varepsilon=0.87$, the o-Ps lifetime-to-hole size relationship cannot calculate the o-Ps lifetimes correctly for this polymeric system. With respect to the evaluation of the cavity shape we suggest the cylindrical model is optimal for estimating the o-Ps lifetime.

\section{E. The cavity environment}

In this section we investigate the free-volume environment of the cavities. The computed cavity-cavity radial distribution functions [Fig. 6(a)], show some structured features for more sensitive probes (below $R_{P}=0.70 \AA$ ). The peaks at close distance are not observed for large probes, implying, that there is some subtle structural feature sampled with the
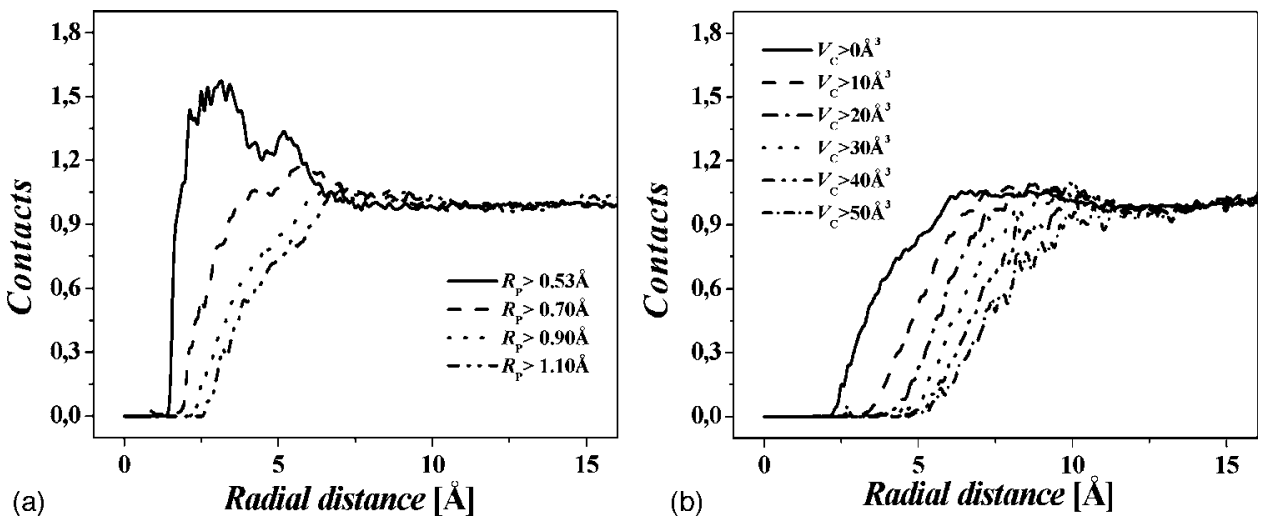

FIG. 6. The cavity-cavity radial distribution function computed (a) for different probe radii $R_{P}$ (b) for different threshold cavity volumes $V_{C}$. 
smaller probes. A similar feature has been observed previously, ${ }^{67}$ when a prepeak on cavity-cavity structure factors was identified as a result of chemical ordering in a simple model for monatomic liquids. The prepeak vanished with increasing the probe radii. Here we observed that excluding the cavities with a certain smaller volume has a similar effect on the distribution functions as the increasing of the probe radius [Fig. 6(b)]. This implies a random distribution of large cavities in the system. We suggest that from the point of view of the PALS two kinds of the free volume are present. The above described structure featured peaks suggest a reminiscence of the chemical structure of the polymeric chain. Since they are related to cavities smaller than the observed cavity threshold volume, most likely these cavities will be forbidden for the o-Ps localization. The PALS can see mainly what we term here the 'bulk free volume', which is described by unstructured cavity-cavity distribution functions computed on cavities with larger volumes.

\section{CONCLUSIONS}

We have performed a computational study of the free volume in the simulated structures of a polymeric system PVME at $300 \mathrm{~K}$. The structures were prepared by means of long MD simulation runs, while a very good agreement between the simulated and experimental densities, with a relative deviation of about $0.7 \%$, has been obtained. The free volume of the structure was analyzed by means of several recently developed methods. The distribution of the number of cavities with the probe radii has shown a characteristic peak at scale comparable with the typical radius of the o-Ps particle. On the other hand, the cavity structure has been found to be percolated when probed by a probe with the o-Ps radius. The structure of the percolated cavity has been redistributed into cavities around the local maxima of distances from the atoms. This approach gives a good agreement of the o-Ps lifetime with the experimental value. This agreement is found when one assumes the coexistence of several o-Ps particles in the percolated cavity structure and rejects the possibility of o-Ps annihilating in the small cavities. The best approximation of cavity shape was numerically evaluated for the ellipsoidal geometry. The number average of the enumerated volume overlap was $84.4 \%$; however, for the volume weighted average the value decreased to $68.5 \%$ because of the increasing deviation of regularities with increasing volume of cavities. The ellipsoidal model was not suitable for this kind of system because of the high eccentricity of the cavities. Thus, the cylindrical-like approximation appears to be the most appropriate one for estimation of the o-Ps lifetime. The cavity-cavity distribution functions showed a structured feature that disappeared for larger cavities and was not observed when larger probes were used. This may indicate that for the PALS mainly the "bulk free volume" with a random occurrence is important.

\section{ACKNOWLEDGMENTS}

This work was supported by the VEGA Agency, Slovakia under Grant No. 2/0014/09 (J.B.) and Project No. MAT2007-63681 (Spanish Ministry of Education), Grant
No. IT-436-07 (Basque Government) and Project No. 2007SK0008 between Slovak Academy of Sciences (SAS) and Spanish Research Council (CSIC). Support from the project Spanish MEC Grant No. CSD2006-53 is also acknowledged.

${ }^{1}$ A. J. Batschinski, Z. Phys. Chem. 84, 643 (1913).

${ }^{2}$ T. G. Fox and P. J. Flory, J. Appl. Phys. 21, 581 (1950).

${ }^{3}$ A. K. Doolittle, J. Appl. Phys. 22, 1031 (1951).

${ }^{4}$ T. G. Fox and P. J. Flory, J. Phys. Chem. 55, 211 (1951).

${ }^{5}$ M. L. Williams, R. F. Landel, and J. D. Ferry, J. Am. Chem. Soc. 77, 3701 (1955).

${ }^{6}$ D. H. Cohen and D. Turnbull, J. Chem. Phys. 31, 1164 (1959).

${ }^{7}$ D. Turnbull and M. H. Cohen, J. Chem. Phys. 34, 120 (1961).

${ }^{8}$ D. Turbull and M. H. Cohen, J. Chem. Phys. 52, 3038 (1970).

${ }^{9}$ G. S. Grest and M. H. Cohen, Adv. Chem. Phys. 48, 455 (1981).

${ }^{10} \mathrm{~W}$. Brandt and A. Dupasquier, Positron Solid State Physics (North-Holland, Amsterdam, 1983).

${ }^{11}$ Y. C. Jean, Microchem. J. 42, 72 (1990).

${ }^{12}$ Y. C. Jean, in Positron Spectroscopy of Solids, edited by A. Dupasquier (IOS, Ohmsha, Amsterdam, 1995), pp. 563-580.

${ }^{13}$ J. Bartoš, in Encyclopedia of Analytical Chemistry, edited by R. A. Meyers (Wiley, New York, 2000), pp. 7968-7987.

${ }^{14}$ Principles and Applications of Positron \& Positronium Chemistry, edited by Y. C. Jean, P. E. Mallon, and D. M. Schrader (World Scientific, Singapore, 2003).

${ }^{15}$ J. Bartoš, D. Račko, O. Šauša, and J. Krištiak, Soft Matter under Exogenic Impacts, ARW NATO Series (Springer, New York, 2007), p. 113.

${ }^{16}$ G. Dlubek, in Encyclopedia of Polymer Science and Technology, edited by A. Seidel (Wiley, New York, 2008).

${ }^{17}$ S. J. Tao, J. Chem. Phys. 56, 5499 (1972).

${ }^{18}$ M. Eldrup, D. Lightbody, and J. N. Sherwood, Chem. Phys. 63, 51 (1981).

${ }^{19}$ H. Nakanishi, S. J. Wang, and Y. C. Jean, in Positron Annihilation Studies of Fluids, edited by S. C. Sharma (World Science, Singapore, 1988).

${ }^{20} \mathrm{~T}$. Goworek, J. Nucl. Radiochem. Sci. 1, 11 (2000).

${ }^{21}$ Y. C. Jean and H. Shi, J. Non-Cryst. Solids 172-174, 806 (1994).

${ }^{22}$ Y. C. Jean, H. Shi, G. H. Dai, C. M. Huang, and J. Liu, Mater. Sci. Forum 175-178, 691 (1995).

${ }^{23}$ Y. C. Jean, Y. Rhee, Y. Lou, D. Shelby, and G. L. Wilkes, J. Polym. Sci., Part B: Polym. Phys. 34, 2979 (1996).

${ }^{24}$ B. Jasinska, A. E. Koziol, and T. Goworek, J. Radioanal. Nucl. Chem. 210, 617 (1996).

${ }^{25}$ D. W. Gidley, W. E. Frieze, T. L. Dull, A. F. Yee, E. T. Ryan, and H.-M. Ho, Phys. Rev. B 60, R5157 (1999).

${ }^{26}$ D. W. Gidley, W. E. Frieze, T. L. Dull, J. Sun, A. F. Yee, C. V. Nguyen, and D. Y. Yoon, Appl. Phys. Lett. 76, 1282 (2000).

${ }^{27}$ J. N. Sun, Y. F. Hu, W. E. Frieze, and D. W. Gidley, Radiat. Phys. Chem. 68, 345 (2003).

${ }^{28}$ B. G. Olson, T. Prodpran, A. M. Jamieson, and S. Nazarenko, Polymer 43, 6775 (2002).

${ }^{29}$ D. Rigby and R. J. Roe, Macromolecules 23, 5312 (1990).

${ }^{30}$ S. Arizzi, P. H. Mott, and U. W. Suter, J. Polym. Sci., Part B: Polym. Phys. 30, 415 (1992).

${ }^{31}$ R. Voorintholt, M. T. Kosters, G. Vegter, G. Vriend, and W. G. J. Hol, J. Mol. Graphics 7, 243 (1989).

${ }^{32}$ E. G. Kim, S. Misra, and W. L. Mattice, Macromolecules 26, 3424 (1993).

${ }^{33}$ S. Lee and W. L. Mattice, Comput. Theor. Polym. Sci. 9, 57 (1999).

${ }^{34}$ R. M. Dammert, S. L. Maunu, F. H. J. Maurer, I. M. Neelov, S. Niemela, F. Sundholm, and C. Wästlund, Macromolecules 32, 1930 (1999).

${ }^{35}$ V. M. Shah, S. A. Stern, and P. J. Ludovice, Macromolecules 22, 4660 (1989).

${ }^{36}$ M. L. Connolly, J. Appl. Crystallogr. 16, 548 (1983).

${ }^{37}$ M. L. Connolly, J. Am. Chem. Soc. 107, 1118 (1985).

${ }^{38}$ C. Nagel, E. Schmidtke, K. Günter-Schade, D. Hofmann, D. Fritsch, T. Strunskus, and F. Faupel, Macromolecules 33, 2242 (2000).

${ }^{39}$ C. Nagel, K. Günter-Schade, D. Fritsch, T. Strunskus, and F. Faupel, Macromolecules 35, 2071 (2002).

${ }^{40}$ D. Hofmann, M. Heuchel, Y. Yampolskii, V. Khotimskii, and V. Shantarovich, Macromolecules 35, 2129 (2002).

${ }^{41}$ D. Hofmann, M. Entrialgo-Castano, A. Lerbret, M. Heuchel, and Y. Yampolskii, Macromolecules 36, 8528 (2003). 
${ }^{42}$ M. Heuchel, D. Hofmann, and P. Pullumbi, Macromolecules 37, 201 (2004).

${ }^{43}$ E. Schmidtke, K. Gunter-Schade, D. Hofmann, and F. Faupel, J. Mol. Graphics Modell. 22, 309 (2004).

${ }^{44}$ K. Šolc, Polym. News 4, 67 (1977).

${ }^{45}$ D. N. Theodorou and U. W. Suter, Macromolecules 18, 1206 (1985).

${ }^{46}$ J. Bartoš, J. Urban, P. Mach, and J. Krištiak, Mater. Sci. Forum 363-365, 294 (2001).

${ }^{47}$ D. Račko, R. Chelli, G. Cardini, J. Bartoš, and S. Califano, Eur. Phys. J. D 32, 289 (2005).

${ }^{48}$ D. Račko, R. Chelli, G. Cardini, S. Califano, and J. Bartoš, Theor. Chem. Acc. 118, 443 (2007).

${ }^{49}$ S. W. Bunte and H. Sun, J. Phys. Chem. B 104, 2477 (2000).

${ }^{50}$ H. Sun, J. Phys. Chem. B 102, 7338 (1998).

${ }^{51}$ J. Yang, Y. Ren An-min Tian, and H. Sun, J. Phys. Chem. B 104, 4951 (2000).

${ }^{52}$ J. Colmenero, F. Alvarez, and A. Arbe, Phys. Rev. E 65, 041804 (2002).

${ }^{53}$ A. Bondi, J. Phys. Chem. 68, 441 (1964).

${ }^{54}$ J. Higo and N. Go, J. Comput. Chem. 10, 376 (1989).

${ }^{55}$ LAPACK driver routine (version 3.0), University of Tennessee, University of California Berkeley, NAG Ltd., Courant Institute, Argonne National Lab, and Rice University (1999).
${ }^{56}$ A. I. Borisenko and I. E. Tarapov, Vector and Tensor Analysis with Applications (Dover, New York, 1979).

${ }^{57}$ L. Euler, Opera Omnia, Ser. 1, "Commentationes Arithmeticae (Teubner, Berlin, 1917), Vol. XV, p. 217.

${ }^{58}$ T. Shiomi, F. Hamada, T. Nasako, K. Yoneda, K. Imai, and A. Nakajima, Macromolecules 23, 229 (1990).

${ }^{59}$ S. Sastry, T. M. Truskett, P. G. Debenedetti, S. Torquato, and F. H. Stillinger, Mol. Phys. 95-2, 289 (1998).

${ }^{60}$ J. L. Finney, Proc. R. Soc. London Ser. A 319, 479 (1970).

${ }^{61}$ W. Brostow, J. P. Dussault, and B. L. Fox, J. Comput. Phys. 29, 81 (1978).

${ }^{62}$ M. Tanemura, T. Ogawa, and N. Ogita, J. Comput. Phys. 51, 191 (1983).

${ }^{63}$ N. N. Medvedev, J. Comput. Phys. 67, 223 (1986).

${ }^{64}$ D. T. Lee and B. J. Schachter, Int. J. Comput. Inf. Sci. 9, 219 (1980).

${ }^{65}$ Z. Yu, Ph.D. thesis, Case Western Reserve University, 1995.

${ }^{66}$ J. Bartoš, V. Majerník, M. Iskrová, D. Cangialosi, A. Alegría, O. Šauša, A. Arbe, J. Colmenero, and J. Krištiak, "The temperature dependent PALS measurements in PVME-Comparison to relaxation and neutron scattering data" (unpublished).

${ }^{67}$ B. Sadigh, M. Dzugutov, and S. R. Eliot, Phys. Rev. B 59, 1 (1999). 